\title{
Stapedial reflex in amyotrophic lateral sclerosis
}

\author{
Toshio Shimizu, Tetsuro Hayashida, Hideaki Hayashi, Shuichi Kato, Hitoshi Tanabe
}

Department of Neurology, Tokyo Metropolitan Neurological Hospital, Tokyo, Japan T Shimizu $S$ Kato

H Tanabe

Department of Neurootology, Tokyo Metropolitan Neurological Hospital, Tokyo, Japan T Hayashida

Department of Neurology, Tokyo Metropolitan Komagome Hospital, Tokyo, Japan H Hayashi

Correspondence to: Dr Toshio Shimizu, Department of Neurology, Tokyo Metropolitan Neurological Hospital, 2-6-1 Musashidai, Fuchu, Tokyo 183, Japan.

Received 12 July 1995 and in revised form 3 December 1995 Accepted 12 January 1996

\begin{abstract}
Objective-To examine mechanisms controlling the stapedial reflex in patients with amyotrophic sclerosis (ALS).

Methods-The stapedial reflex was examined using impedance audiometry in 38 patients with sporadic ALS and in 25 age matched controls.

Results-All patients showed normal reflex decay test results. There were no significant differences between patients with ALS and control subjects in reflex threshold, latency, amplitude, or contraction time $\left(C_{50}\right)$. Although each reflex variable in the patients with classic or progressive muscular atrophy types of ALS showed no significant difference from that in control subjects, the patients with bulbar type ALS showed significantly longer latency, $C_{50}$, and retraction time $\left(D_{50}\right)$, and significantly lower amplitude than control subjects. Three types of abnormal reflex waveforms (polyphasic, abnormally delayed retraction, and abnormally early retraction) were noted in six patients.

Conclusion-The subclinical involvement of the stapedius motor neurons or of the supranuclear stapedius motor system might be responsible for the abnormalities of the stapedial reflex in ALS.
\end{abstract}

(F Neurol Neurosurg Psychiatry 1996;60:544-548)

Keywords: amyotrophic lateral sclerosis; stapedial reflex; impedance audiometry; stapedius motor neuron; bulbar palsy introduction

Amyotrophic lateral sclerosis (ALS) is a neurodegenerative disease involving the motor neuron system. In ALS, the involvement of the main facial motor neurons innervating facial mimetic muscles has been confirmed clinicopathologically, but that of the stapedius motor neurons running in the facial nerves

Table 1 Reflexes in patients with ALS

\begin{tabular}{|c|c|c|c|c|}
\hline & & $\begin{array}{l}\text { faw reflex } \\
(n(\%))\end{array}$ & $\begin{array}{l}\text { DTR in upper limbs } \\
(n(\%))\end{array}$ & $\begin{array}{l}\text { DTR in lower limbs } \\
(n(\%))\end{array}$ \\
\hline ALS $(n=38)$ & $\begin{array}{l}\text { Exaggerated } \\
\text { Diminished }\end{array}$ & $\begin{array}{r}22(57.9) \\
6(15.8)\end{array}$ & $\begin{array}{l}18(47 \cdot 4) \\
18(47 \cdot 4)\end{array}$ & $\begin{array}{r}29(76 \cdot 3) \\
7(18 \cdot 4)\end{array}$ \\
\hline Classic $(n=20)$ & Exaggerated & $13(65 \cdot 0)$ & $14(70 \cdot 0)$ & $20(100 \cdot 0)$ \\
\hline & Diminished & $1(5 \cdot 0)$ & $6(30 \cdot 0)$ & $0(0 \cdot 0)$ \\
\hline Bulbar $(n=13)$ & Exaggerated & $8(61 \cdot 5)$ & $4(30 \cdot 8)$ & $9(69 \cdot 2)$ \\
\hline PMA $(n=5)$ & $\begin{array}{l}\text { Diminished } \\
\text { Exaggerated }\end{array}$ & $\begin{array}{l}3(23 \cdot 1) \\
1(20 \cdot 0)\end{array}$ & $\begin{array}{l}7(53.8) \\
0(0.0)\end{array}$ & $\begin{array}{l}2(15 \cdot 4) \\
0(0 \cdot 0)\end{array}$ \\
\hline & Diminished & $2(40 \cdot 0)$ & $5(100 \cdot 0)$ & $5(100 \cdot 0)$ \\
\hline
\end{tabular}

PMA = progressive muscular atrophy; DTR = deep tendon reflexes.

and innervating the stapedius muscles has not been established. In the present study, to analyse impairment of the stapedius motor system clinically, the stapedial reflex-which physiologically protects the inner ear against exposure to hazardous sound by contracting the stapedius muscles in the middle ear-was investigated by impedance audiometry in patients with ALS.

\section{Materials and methods \\ SUBJECTS}

Thirty eight patients with sporadic ALS were studied (26 men, 12 women; mean age 56.9 (SD) 11.3 years (range $34.2-82.2$ years); mean duration of the disease $2.8(2.9)$ years (range 0.3-11.4 years)). In addition, 25 control subjects showing no clinical signs of brainstem, peripheral facial nerve, or cochlear nerve dysfunction (15 men, 10 women; mean age 57.9 (SD) 12.2 years; range $32.3-72.3$ years) were selected. Sex and age were matched between the two groups. The diagnosis of ALS was based on clinical features and results of neurophysiological evaluation. All patients showed involvement of systemic and selective voluntary motor neuron systems with no abnormal signs in the sensory, cerebellar, or extrapyramidal systems. According to the conventional classification of ALS based on predominant signs in the early stage, 20 patients (14 men and six women) were designated as classic type, showing predominant signs of amyotrophy and upper motor neuron involvement; 13 patients (nine men and four women) as bulbar type, showing predominant signs of corticobulbar and bulbar involvement with mild upper and lower corticospinal impairment; and five patients (three men and two women) as progressive muscular atrophy type, showing predominant signs of lower motor neuron involvement in the limbs. ${ }^{12}$ The mean (SD) ages at the time of examination in the subgroups were $55 \cdot 2(8.5), 61.0(14 \cdot 2)$, and $53 \cdot 1(12 \cdot 2)$ years, respectively. The mean (SD) durations of the disease in the subgroups were $3.3(3.0), 1 \cdot 4(0.8)$, and $4.6(4.4)$ years respectively.

Table $i$ summarises the jaw reflexes and deep tendon reflexes in the limbs in the patients examined around the time of the stapedial reflex investigation. Patients with normal reflexes are not included. The degree of the reflexes was judged from physical examination at the bedside. The frequency of the classic type patients with exaggerated jaw reflexes was similar to that of the bulbar type. 
Figure 1 Reflex variables measured in the present study. $A=$ amplitude; $L=$ latency.

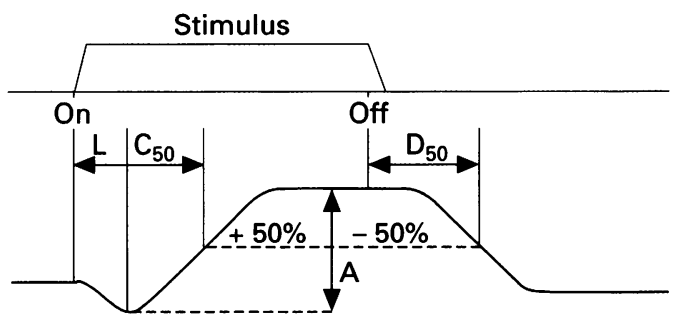

Figure 2 Stapedial reflex thresholds in control subjects and in patients with $A L S$. The threshold values in each patient and the mean (SD) in each group are shown. $P M A=$ progressive muscular atrophy.

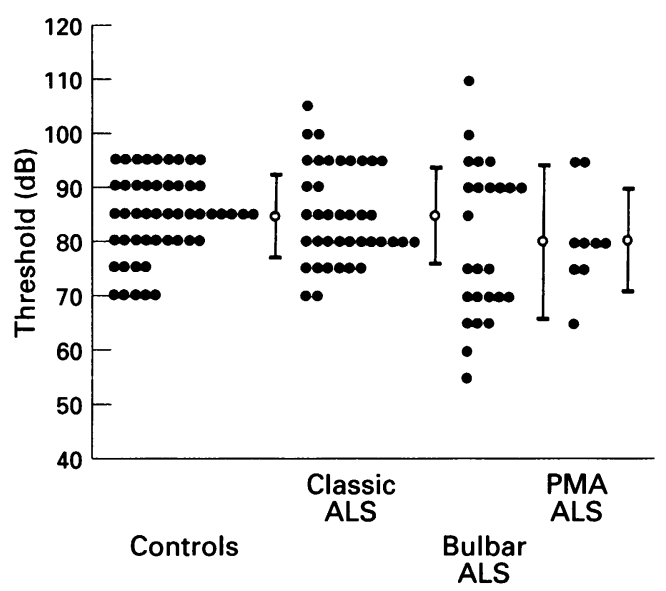

The deep tendon reflexes in the classic type patients were exaggerated more often than those in the bulbar type patients. Only one patient with the progressive muscular atrophy type had an exaggerated jaw reflex, but he was classified into the progressive muscular atrophy type because his deep tendon reflexes were constantly diminished from the disease onset. All the patients with progressive muscular atrophy type had diminished deep tendon reflexes.

Each subject showed a hearing sensitivity of less than $30 \mathrm{~dB}$ hearing level $(\mathrm{HL})$ at $500 \mathrm{~Hz}$, normal tympanogram (type $\mathrm{A}$ ), and the presence of a stapedial reflex at $500 \mathrm{~Hz}$.

\section{APPARATUS}

Measurements of the stapedial reflex were performed with an oto-reflex analyser model RS06 (RION Co Ltd, Japan), and a videoprinter model RE-20 (RION Co Ltd, Japan) was used for recording.

\section{PROCEDURE}

Initially pure tone air conduction thresholds at 500,1000 , and $2000 \mathrm{~Hz}$ were examined by standard procedures in a double walled soundproof room, and tympanograms were obtained. In each subject, stapedial reflex thresholds were detected in each ear by presenting a $500 \mathrm{~Hz}$ pure tone stimulus contralaterally. The reflex decay test was carried out with a pure tone stimulus of $500 \mathrm{~Hz}$ at $10 \mathrm{~dB}$ above the reflex threshold. The stimulus duration was 10 seconds in the decay test. A reduction of the response amplitude of more than $50 \%$ during the 10 second stimulation was regarded as a positive result in the reflex decay test.

To measure several reflex variables, 10 sequential reflexes were averaged and recorded using pure tone stimuli of $500 \mathrm{~Hz}$ at $10 \mathrm{~dB}$ above the reflex threshold. The stimulus duration was $500 \mathrm{~ms}$ and the rise/fall time was $10 \mathrm{~ms}$. The analysis time was 1 second, and the interstimulus interval was 1.5 seconds. Reflex variables were measured as follows (fig 1): latency = time from the onset of the stimulus to the first increase in acoustic impedance; contraction time $\left(\mathrm{C}_{50}\right)=$ time from the first increase in acoustic impedance to $50 \%$ of maximum amplitude; retraction time $\left(D_{50}\right)=$ time from the cessation of the stimulus to the point when amplitude had decreased to $50 \%$ of maximum. Amplitude was defined as the difference between the highest and the lowest value of impedance, and was expressed in arbitrary units (au).

\section{STATISTICAL ANALYSIS}

The mean (SD) values of threshold,latency, $\mathrm{C}_{50}, \mathrm{D}_{50}$, and amplitude were calculated in both groups and in each subgroup of patients with ALS. The data were compared between each group with the Mann-Whitney $U$ test. The $P$ values for all data were two sided, and the level of significance was set at $5 \%$.

\section{FACIAL EMG}

Facial needle EMG was performed on 10 patients with classic type, nine with bulbar type, and five with progressive muscular atrophy type. We examined EMG neurogenic changes such as reduced interference pattern, motor unit potentials with high amplitude and long duration, and polyphasic motor unit potentials in the orbicularis oculi or orbicularis oris muscle according to standard criteria. ${ }^{3}$

\section{Results}

HEARING LEVEL

The mean (SD) hearing level at $500 \mathrm{~Hz}$ was $14.6(6.4) \mathrm{dB}$ HL in the control subjects and $15 \cdot 4(5 \cdot 6) \mathrm{dB} \mathrm{HL}$ in the patients with ALS. The mean (SD) hearing levels at $500 \mathrm{~Hz}$ for the subgroups of patients were $16.3(4.9) \mathrm{dB}$ HL for the classic type, $14.4(6.0) \mathrm{dB}$ HL for the bulbar type, and $14.5(6 \cdot 9) \mathrm{dB}$ HL for the progressive muscular atrophy type. No significant differences were seen between the patient and the control values.

The mean (SD) hearing level at 500, 1000, and $2000 \mathrm{~Hz}$ was $16.0(9.7) \mathrm{dB} \mathrm{HL}$ in the control subjects and $16 \cdot 0(7 \cdot 5) \mathrm{dB} \mathrm{HL}$ in the

Table 2 Reflex threshold and variables in control subjects and in patients with ALS

\begin{tabular}{|c|c|c|c|c|c|}
\hline & Threshold (dB) & Lalency (ms) & Amplitude (au) & $C_{s 0}(m s)$ & $D_{s o}(m s)$ \\
\hline $\begin{array}{l}\text { Control }(n=25) \\
\text { ALS }(n=38) \\
\text { Classic }(n=20) \\
\text { Bulbar }(n=13) \\
\text { PMA }(n=5)\end{array}$ & $\begin{array}{l}84 \cdot 5(7 \cdot 6) \\
82 \cdot 7(11 \cdot 2) \\
84 \cdot 9(8 \cdot 9) \\
80 \cdot 2(14 \cdot 3) \\
80 \cdot 6(9 \cdot 5)\end{array}$ & $\begin{array}{l}77 \cdot 7(13 \cdot 9) \\
82 \cdot 0(22 \cdot 0) \\
78 \cdot 0(22 \cdot 0) \\
88 \cdot 0(24 \cdot 3)^{\star} \\
82 \cdot 6(12 \cdot 0)\end{array}$ & $\begin{array}{l}27 \cdot 9(16 \cdot 9) \\
23 \cdot 5(15 \cdot 0) \\
26 \cdot 5(15 \cdot 1) \\
17 \cdot 4(12 \cdot 1)^{\star \star \star} \\
27 \cdot 3(17 \cdot 4)\end{array}$ & $\begin{array}{c}81 \cdot 7(37 \cdot 0) \\
93 \cdot 4(40 \cdot 0) \\
81 \cdot 1(35 \cdot 5) \\
114 \cdot 8(42 \cdot 8)^{\star \star \star} \\
87 \cdot 5(28 \cdot 2)\end{array}$ & $\begin{array}{l}236 \cdot 8(91.9) \\
266 \cdot 3(94.5)^{\star} \\
262.5(101 \cdot 7) \\
273.2(75 \cdot 2)^{\star} \\
258 \cdot 9(128 \cdot 8)\end{array}$ \\
\hline
\end{tabular}

Values are means (SD); PMA $=$ progressive muscular atrophy; ${ }^{\star} P<0.05 ;{ }^{\star \star \star} P<0.001$. 

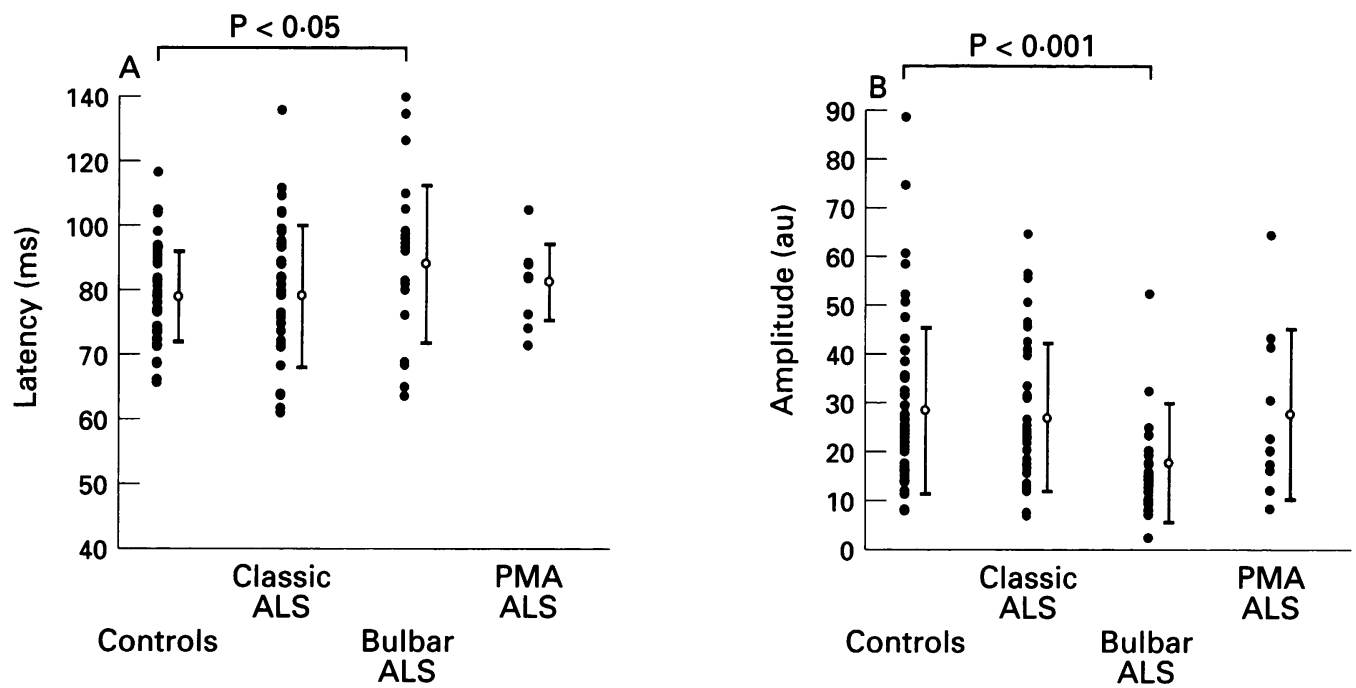

Figure 3 Latency $(A)$ and amplitude $(B)$ of the averaged stapedial reflex in control subjects and in patients with ALS. The values for each patient and the mean (SD) for each group are shown. PMA = progressive muscular atrophy.

patients with ALS. For the subgroups of patients, it was $16 \cdot 1(5 \cdot 5) \mathrm{dB}$ HL for the classic type, $15.5(8.6) \mathrm{dB}$ HL for the bulbar type, and $17 \cdot 1(10.9) \mathrm{dB} \mathrm{HL}$ for the progressive muscular atrophy type. None of these values were significantly different from controls.

\section{REFLEX THRESHOLD}

The mean (SD) reflex threshold of 84.5 (SD $7 \cdot 6$ ) $\mathrm{dB} \mathrm{HL}$ (range 70-95 dB HL) in the control subjects was not significantly different from that of 82.7 (SD 11.2) dB HL (range 55-1 $10 \mathrm{~dB}$ HL) in the patients with ALS. The mean (SD) reflex thresholds for the subgroups of patients with ALS were $84.9(8.9) \mathrm{dB}$ in the classic type, $80.2(14.3) \mathrm{dB}$ in the bulbar type, and $80.6(9.5) \mathrm{dB}$ in the progressive muscular atrophy type (fig 2). There was no significant difference between the reflex threshold in the control subjects and that in each subgroup of patients with ALS.

\section{REFLEX DECAY TEST}

All control subjects and all patients with ALS showed negative reflex decay test results.

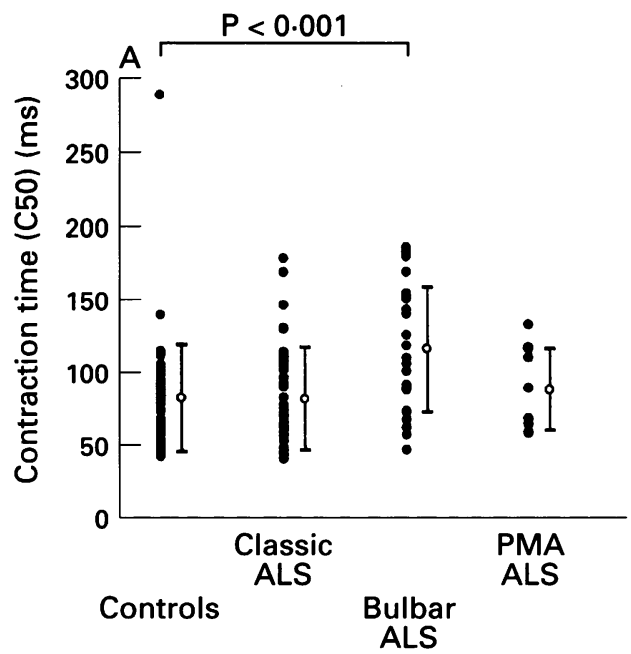

\section{REFLEX VARIABLES}

Table 2 shows each reflex variable in the control subjects and in the patients with ALS. $D_{50}$ in the patients $(266.3$ (SD 94.5) ms) was significantly longer than that in the control subjects (236.8 (SD 91.9) $\mathrm{ms} ; \quad P<0.05)$, but there were no significant differences between latency, $\mathrm{C}_{50}$, and amplitude in the control subjects and those in the patients with ALS.

Table 2 and figs 3 and 4 show the reflex variables in each subgroup of patients. In the patients with bulbar type ALS, latency, $\mathrm{C}_{50}$, and $D_{50}$ were significantly longer than those in the control subjects $(P<0.05, P<0.001$, and $P<0.05$ respectively), and amplitude was significantly lower than that in the control subjects $(\mathbf{P}<0.001)$. In the patients with classic and progressive muscular atrophy type ALS, the reflex variables showed no significant differences compared with controls.

In the control subjects, the averaged reflex waveform was usually monophasic or diphasic with or without an initial dip (fig 5A). A reflex waveform with four or more positive impedance peaks was considered abnormal. Three

Figure 4 Contraction time $(A)$ and retraction time $(B)$ of the averaged stapedial reflex in control subjects and in patients with ALS. The values for each patient and the mean (SD) for each group are given. PMA = progressive muscular atrophy.

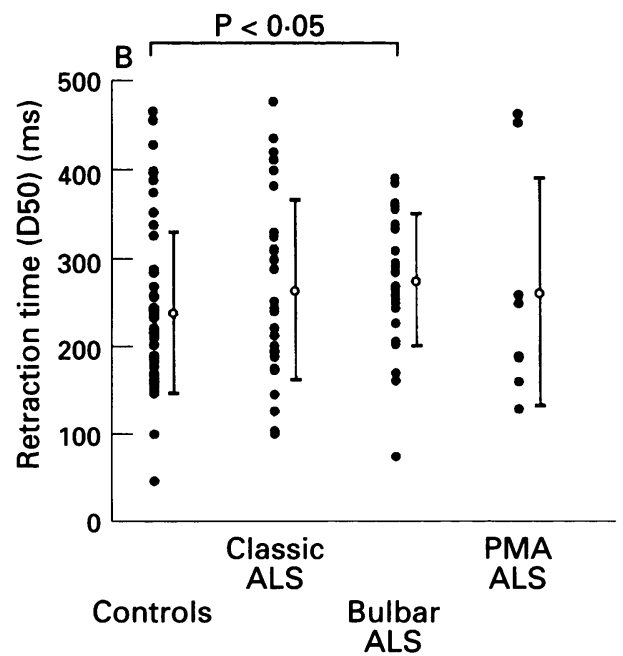


Figure 5 Typical waveforms of the stapedial reflex. (A) Normal reflex waveform in a control subject. (B) Polyphasic or unstable reflex waveform in a patient with bulbar type ALS (a 72 year old woman). (C) Reflex waveform with abnormally early retraction - that is, negative $D_{50}$ - in a patien with classic type ALS (a 52 year old man).

Latency, $C_{50}$, and amplitude were within normal ranges. (D) Reflex waveform with abnormally delayed retraction in a patient with classic type ALS (a 70 year old man). $D_{s 0}$ could not be measured within the analysis time of 1.5 seconds. Vertical bar $=$ 10 au, horizontal bar $=$ $100 \mathrm{~ms}$.
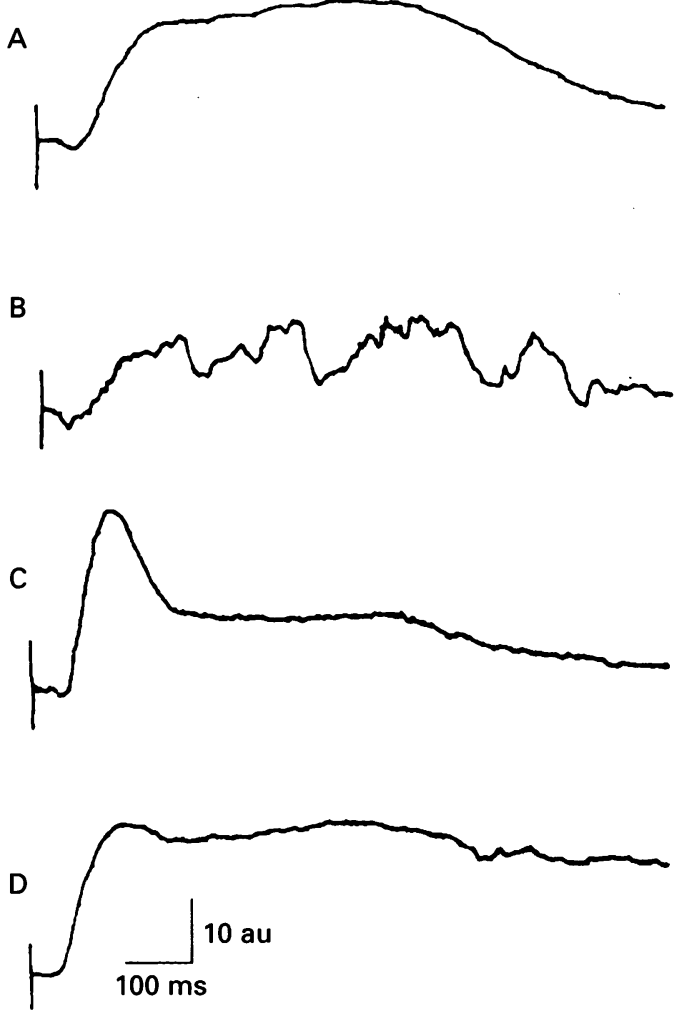

patients with ALS (two with classic type and one with bulbar type) showed polyphasic or unstable averaged reflex waveforms (fig 5B). In two other patients with classic type ALS, another type of abnormal reflex waveform was seen (fig 5C). Although latency, $C_{50}$, and amplitude were within normal ranges in those patients, the maximum gain of reflex was not maintained before cessation of the acoustic stimulation. Accordingly, $D_{50}$ was negative in those patients. On the other hand, one patient with classic ALS showed abnormally delayed retraction (fig $5 \mathrm{D}$ ). In this patient, $\mathrm{D}_{50}$ could not be measured within the analysis time of 1.5 seconds. In the remaining 32 patients, the averaged reflex waveforms showed a normal pattern.

There was no significant correlation between the reflex threshold or any reflex variables and the duration of the disease in patients with ALS.

\section{FACIAL EMG}

In the facial needle EMG study, seven of 10 patients with classic type, eight of nine bulbar type, and one of three progressive muscular atrophy type showed one or more of the neurogenic changes such as reduced interference pattern, motor unit potentials with high amplitude and long duration, and polyphasic motor unit potentials.

\section{Discussion}

In the evaluation of each reflex variable, the patients with bulbar type showed the following abnormal findings: prolongation of latency, $\mathrm{C}_{50}$, and $\mathrm{D}_{50}$, and decrease of amplitude. The prolongation of the mean $D_{50}$ in all 38 patients is considered to reflect mainly the prolonga- tion of $\mathrm{D}_{50}$ in the patients with bulbar type, because those in the patients with classic and progressive muscular atrophy type were within the normal ranges. Possible candidates for the lesion responsible for these abnormalities are (a) the stapedius motor neurons, (b) the interneurons in the brainstem, and (c) the higher regulating system or the corticofugal tract projecting to the reflex arc.

Because amplitude is known to be the most sensitive indicator of neuropathological processes among the reflex variables, and latency usually increases as amplitude decreases, ${ }^{4}$ the prolongation of latency and $\mathrm{C}_{50}$ may be a reflection of the decreased amplitude. Generally in the limb muscles of patients with ALS, axonal degeneration of motor neurons induces slightly slowed latency and reduced amplitude of compound muscle action potentials. ${ }^{5}$ The prolongation of latency and $\mathrm{C}_{50}$ and the decrease of amplitude may be explained by subclinical involvement of the stapedius motor neurons. Moreover, the polyphasic or unstable reflex waveforms seen in three cases (fig 5B) might be due to insufficient muscle contraction and resultant unstable membrane deflection.

The prolongation of relaxation time of the stapedial reflex has been reported in various neuromuscular disorders, ${ }^{6-9}$ but its mechanism remains controversial. The stapedial reflex is mediated by interconnected neural pathways in the brainstem. A direct pathway of the reflex involves neurons in the ventral cochlear nucleus with their axons running in the trapezoid body, and interneurons in the medial superior olivary complex. ${ }^{1011}$ Borg $^{10}$ also described a multisynaptic indirect pathway of the reflex and proposed the lateral zone of the reticular formation as the responsible region. According to Borg's experiments, a lesion involving the direct pathway increased the latency, delayed the onset, and slightly increased the relaxation time of the stapedial reflex, and these findings were considered to reflect the activities of the indirect pathway. ${ }^{10}$ If interneurons mediating the direct pathway are involved, the contraction and relaxation time might be prolonged. However, no pathological evidence that the trapezoid body or the medial superior olivary complex is involved in ALS has been reported.

Another possible explanation for the prolongation of $D_{50}$ is the involvement of a higher regulating system. Experimental ablation of the auditory cortex or intercollicular decerebration induced disappearance of a long latency component of the acoustic reflex EMG. ${ }^{12}{ }^{13}$ Furthermore, voluntary movements of the laryngeal and pharyngeal muscles have been reported to increase stapedius muscle activity. ${ }^{14} 15$ These findings suggest corticofugal descending regulation from the auditory cortex and cortical area which integrates motor behaviour. If the descending inhibitory regulation is impaired, the acoustic reflex might be delayed, and the relaxation time prolonged.

The early retraction seen in two patients (fig 5C) can be explained by the involvement of the indirect pathway in the medullary reticular 
formation or of the descending regulating system. The appearance of Bunina bodies in the medullary reticular formation in ALS reported by Nakano et al $^{16}$ suggests the possibility of indirect pathway involvement and abolishment of the long latency component of the stapedial reflex. On the other hand, if the descending excitatory projection to the reflex arc is impaired, the late component of the stapedius reflex can be lost, resulting in early relaxation of the stapedius muscles. Disappearance of the R2 component despite the presence of a normal R1 component in studies of the blink reflex in ALS or cerebrovascular disease also suggests supranuclear dysregulation. ${ }^{17} 18$

Why did only the patients with bulbar type show the abnormal reflex variables in this study? As the facial mimetic muscles are usually affected in both bulbar and classic types of ALS, it cannot be explained by the degree of facial muscle involvement. The needle EMG findings also disclosed no differences in the frequency of the EMG neurogenic changes of the facial muscles between the bulbar type and the classic type. Moreover, it is unlikely that the distortion of facial musculature and the resultant change of the middle ear bone compliance caused the reflex abnormalities in the bulbar type of ALS, because the tympanograms in all the patients were normal. The significantly decreased amplitude in the bulbar type suggests that the stapedius motor neurons are more vulnerable in the bulbar type than in the other subgroups of ALS. In addition, the degeneration of the stapedius motor neurons may probably be independent of that of the facial mimetic motor neurons in ALS, a view supported by the anatomical findings that the stapedius motor neurons are located in the perifacial and the accessory stapedius nuclei distinct from the main facial nucleus. ${ }^{19-21}$ Because the frequency of the bulbar type patients with exaggerated jaw reflexes or deep tendon reflexes was similar to or less than that of the classic type patients in this study (table 1), the degenerations of the corticotrigeminal tract and the corticospinal tract may have no correlation with the stapedial reflex abnormalities in the bulbar type. Salomon and Starr ${ }^{14}$ reported that movements of the laryngeal and pharyngeal muscles were always associated with an increase in stapedial EMG activity, and that, by contrast, movements of the facial muscles such as tight voluntary eye closure were not associated with such activity. There may be a close relation between the bulbar involvement and the vulnerability of the stapedial reflex in ALS, and a common mechanism may be involved both in degeneration of the corticobulbar tract and that of the corticostapedial tract.

1 Norris FH. Adult progressive muscular atrophy and hereditary spinal muscular atrophies. In: Vinken PJ, Bruyn $\mathrm{GW}$, Klawans HL, de Jong JMBV, eds. Handbook of
clinical neurology, diseases of the motor system. Vol 59. clinical neurology, diseases of the motor system. Vol

2 Mitsumoto $\mathrm{H}$. Classification and clinical features of amyotrophic lateral sclerosis. In: Mitsumoto H, Norris FH, eds. Amyotrophic lateral sclerosis. A comprehensive guide to management. New York: Demos Publications, 1994: $1-19$.

3 Kimura J. Types of abnormality. In: Electrodiagnosis in diseases of nerve and muscle: principles and practice. 2nd ed. Philadelphia, FA Davis, 1989:249-74.

4 Stach BA. The acoustic reflex in diagnostic audiology: from Metz to present. Ear Hear 1987;8:36S-42S.

5 Kimura J. Diseases of the motor neuron. In: Electrodiagnosis in diseases of nerve and muscle: principles and practice. 2nd in diseases of nerve and muscle: principles and

6 Yamane M, Nomura Y. Analysis of stapedial reflex in neuromuscular diseases. ORL $\mathcal{F}$ Otorhinolaryngol Relat Spec 1984;46:84-96.

7 Jerger J, Oliver TA, Rivera V, Stach BA. Abnormalities of the acoustic reflex in multiple sclerosis. Am $\mathcal{F}$ Otolaryngol 1986;7:163-76.

8 Lew H, Jordan C, Jerger J, Jankovic J. Acoustic reflex abnormalities in cranial-cervical dystonia. Neurology 1992;42:594-7.

9 Murofushi T, Yamane M, Osanai R. Stapedial reflex in Parkinson's disease. ORL f Otorhinolaryngol Relat Spec 1992;54:255-8.

10 Borg E. On the neuronal organization of the acoustic middle ear reflex. A physiological and anatomical study. Brain Res 1973;49:101-23.

11 Rouiller EM, Capt M, Dolivo M, Ribaupierre FD. Neuronal organization of the stapedius reflex pathways in the rat: a retrograde HRP and viral transneuronal tracing study. Brain Res 1989;476:21-8.

12 Baust W, Berlucchi G. Reflex response to clicks of cat's tensor tympani during sleep and wakefulness and the influence thereon of the auditory cortex. Arch Ital Biol 1964;102:686-712.

13 Salomon G, Starr A. Electromyographic study of click evoked middle ear muscle activity in cats. Int Audiol 1965;4:31-3.

14 Salomon G, Starr A. Electromyography of middle ear muscles in man during motor activities. Acta Neurol Scand 1963;39:161-8.

15 Borg E, Zakrisson J-E. The activity of the stapedius muscle in man during vocalization. Acta Otolaryngol 1975;79: in man

16 Nakano I, Iwatsubo T, Hashizume Y, Mizutani T. Bunina bodies in neurons of the medullary reticular formation in amyotrophic lateral sclerosis. Acta Neuropathol 1993;85: $471-4$.

17 Kimura J, Wilkinson JT, Damasio H, Adams HR Jr, Shivapour E, Yamada T. Blink reflex in patients with hemispheric cerebrovascular accident (CVA). $¥$ Neurol Sci 1985;67:15-28.

18 Shimoda M, Yokoyama Y, Nakashima K, Takahashi K. Electrically induced blink reflex in patients with amyotrophic lateral sclerosis. Ipn f EEG EMG 1993;21: 337-41. (In Japanese with English abstract.)

19 Joseph MP, Guinan J, Fullerton BC, Norris BE, Kiang NYS. Number and distribution of stapedius motoneurons in cats. F Comp Neurol 1985;232:43-54.

20 Thompson GC, Igarashi M, Stach BA. Identification of stapedius muscle motoneurons in squirrel monkey and stapedius muscle motoneurons in squirrel
bush baby. $₹$ Comp Neurol 1985;231:270-79.

21 Guinan JJ, Joseph MP, Norris BE. Brainstem facial-motor pathways from two distinct groups of stapedius motoneurons in the cat. $\mathcal{F}$ Comp Neurol 1989;287:134-44. 